\title{
Data Communication in Mobile Wireless Sensor Networks
}

\author{
Venkatramana Bhat P. \\ Professor \& HOD \\ Dept of C S\& Engineering, \\ VCET Puttur, Karnataka, India
}

\author{
Shridhar Aithal \\ Professor TAPMI, Manipal \\ Manipal University, \\ Manipal Karnataka, India
}

\author{
Sudhakara Adiga \\ Professor, MIT \\ Manipal University, \\ Manipal Karnataka, India
}

\begin{abstract}
A Mobile Wireless Sensor Network (M-WSN) derives its name by considering either mobile sink or mobile sensor nodes within the Wireless Sensor Network (WSN). As the sensor nodes are energy constrained, energy efficiency is the main aspect to be considered in any applications. By considering mobile sensor nodes in WSN, we can have better energy efficiency, improved coverage, and enhanced target tracking in Wireless Sensor Network. Due to mobility of nodes, a mobile WSN has dynamic topology. For all data gathering applications, the topology of mobile WSN depends on either the path of mobile sink or position of mobile nodes. Hence whole WSN topology keeps on changing as either sink is mobile or sensor nodes are mobile. That is, we have dynamic topology. Depending on application scenario, we may use a mobile sink to collect information from a static WSN or a dynamic WSN. In general static WSN uses multihops for data communication from sensor node to sink. Hence sensor node closer to sink is always in use and its energy gets exhausted quickly, thereby it dies down first, breaking link to sink and whole network collapses. This is one of the serious problems to be considered. Mobile WSN is one of approach that can increase life time of network because nodes close sink keeps on changing so that no particular node will be always close to sink. It is also possible by controlled mobility of nodes, all nodes in turn can take role of being close to sink and provide necessary services. Also by providing mobility to nodes in controlled manner it also possible to reduce number of hops to sink from a node, there by errors in communication gets reduced. In this article we consider two general application areas, studying the conditions of disastrous area where in static sensor nodes are deployed in disastrous area and a mobile sink agent which is outside the boundary moves around predefined path to gather information's of disastrous area, a battle field where in two way data communication between captain and soldiers is required where both captain and soldiers may have low mobility. The networking required in both cases is mobile WSN. We propose proper architecture and data communication in these contexts.
\end{abstract}

\section{Keywords:}

Sensor node, Clustering, Mobile Wireless Sensor Network, Dynamic grouping, Leader - Followers - Mediator.

\section{INTRODUCTION}

The sensor nodes are energy constrained and maximum energy consumption is in communication process. Hence an effective approach on communication can conserve energy consumption thereby increase network life time. One of the ways is to design a procedure to get a logical structure for the physically deployed nodes. Thereby we get a proper network topology on which data transfer may be carried out [1]. In case of large scale Wireless Sensor Networks, wherein thousands of sensor nodes are deployed, proper clustering technique [2] will define proper logical structure. This will conserve energy less than simple multihop communication. When clustering is made considering residual energy of sensor nodes, network life time can be increased considerably. Hence the proposal is to design a grouping strategy depending on energy level of the sensor nodes deployed. Then we perform data transfer on that network.

In the applications such as military, target tracking, microelectronic mechanical devices of stamp size which can sense the surrounding media and transport information to control center can be used. In such applications, we may consider mobile nodes which can increase overall life time of network. Due to mobility of nodes, a mobile WSN [3] has dynamic topology. For all data gathering applications, the topology of mobile WSN depends on either the path of mobile sink or position of mobile nodes. While designing the grouping procedure path from group to group should be unambiguous, thereby communication between any nodes should be possible.

In general, the high level design for data communication in wireless sensor networks [4] may be performed by following procedure.

1) For the thousands of nodes deployed in a controlled area, identify source and sink nodes.

2) Starting from source node form groups and stop procedure once sink node is the member of any group. Store the intermediate nodes between sink and source in that logical structure.

3) Forward data from source to sink through those intermediate nodes.

The article is organized into six sections. Grouping system description defining basic grouping procedure is given in second section. The deferent grouping algorithms to support data communication are given in third section. Design is given in section four. Grouping Scenario with an example, simulated results and observations made is given in fifth section. In Section six conclusions drawn is given. The article ends with references.

\section{GROUPING SYSTEM DESCRIPTION}

Grouping structure which we define has group head which we call as leader, members of group as followers and followers that support to link other group as mediators. Hence the nodes involved in the data communication process may be either a group leader or a follower node and some mediator nodes in the logical structure. Hence we name this as Leader-Follower-Mediator (LFM) algorithm. Considering 
different clustering techniques[5][6][7], an energy efficient[8][9]clustering procedure is proposed here.

Large number of Sensor nodes distributed in a controlled area is assumed to have initial energy greater or equal to some upper threshold value. The location of the nodes is found from the location finding system, embedded in the Sensor nodes. The grouping procedure is as follows.

Staring from one of the node $\mathrm{k}$, check energy level of all the nodes within the transmission range of $\mathrm{k}$ (within one hop distance). The node with highest energy level with in visibility range of $\mathrm{k}$ which has residual energy greater than some upper threshold value is declared as Leader of the group. Other nodes located within the visibility range of that leader and with energy level greater than minimum threshold value can be the followers or members of that group. This forms first cluster in the clustering process. We would like to have minimum number of clusters. This can be achieved by non overlapping leaders thereby no two leaders are not in direct communication range to each other. Hence clusters are linked through a follower node which is within range of present cluster and new cluster we are going to form. We call that follower node that links two clusters as mediator node. Hence cluster leader selects one of farthest follower having residual energy above certain threshold value and calls it as mediator and mediator decides next cluster head which is within its transmission range and is neither leader nor a follower of any group. Nodes located within the visibility range of that leader and with energy level greater than minimum threshold value and neither leader nor a follower of any group becomes the followers of that group. The cluster head selects mediator node to continue grouping process. This clustering process is repeated until all nodes are included in groups (In some cases few nodes can become only followers but not within the range of any leader, that cannot participate in grouping because of its lower energy level, they are as good as dead). Any member with in that group has to forward information to its leader. Any such event identified by leader will pass on the information to server. The leader needs a buffer to collect the information from its members. Main server assures the association of individual members through group leader.

The grouping of sensors need to follow a certain algorithm to prove be efficient. This is discussed in the next section.

\section{GROUPING ALGORITHMS}

The sensor nodes have no global identification. Hence our algorithm has to provide identification to every sensor nodes in the grouping process. Hence we consider all nodes have built in GPS or nodes are using some localization algorithms [10][11] so that its location in the deployed area is known to the sensor nodes.

Depending on the different application scenario there can be two major categories of grouping.

1. Both sink and source nodes within defined boundary area where in two ways communication between nodes is possible.

2. Nodes deployed in a restricted area where in a sink may be located outside the boundary of restricted area, but in direct link with at least one node located in the restricted area.

\subsection{Grouping procedure}

3.1.1The LFM algorithm to get LFM tree where both sink and source nodes are within defined boundary area

Table : 1 Control packets and fields

\begin{tabular}{|l|l|}
\hline Control packets & Fields \\
\hline G_request & From Node_id, Message_type, Status \\
\hline N_response & $\begin{array}{l}\text { To address,Location X,Y,Z coordinates, } \\
\text { Residual_energy, Node Status, } \\
\text { Message_type }\end{array}$ \\
\hline L_Select & $\begin{array}{l}\text { From address, id to node, Location X,Y,Z } \\
\text { coordinates, Status, Message_type }\end{array}$ \\
\hline F_Rigistration & $\begin{array}{l}\text { From address, id to node, Location X,Y,Z } \\
\text { coordinates, Node Status, Message_type }\end{array}$ \\
\hline M_Select & To node_id, , Message_type \\
\hline
\end{tabular}

Input: Source node location and Destination node location

// Depending on the residual energy level of nodes, nodes are said to be in active (MODE 2), follower (MODE 1) or dead (MODE 0) mode.

Step 1: Source broadcasts G_Request signal assuming its node_id=1.

Step 2: Nodes which receive that $G_{-}$Request signal will register by sending N_Response signal with nodes residual energy and node location (x, y.z coordinates) to source.

Step 3: If destination location is among responded nodes source becomes Leader and Destination node becomes follower giving node_id to destination by sending F_Registration signal and stop the grouping procedure.

If no nodes among registered nodes have energy level greater than energy level of source, then source itself becomes leader.

Else among nodes responded with source, node having energy level greater than energy level of source and one with highest energy level is selected as Leader, sending L_Select signal assuming leader node_id $=$ node_id and reset node_id of source to 0 . Ties are broken at random.

Step 4: Leader sends G_Request signal, the nodes within range of the leader respond with N_Response signal with their residual energy and location. They are registered as followers of that group, leader allocates node_id (node_id=node_id+1) to its followers by sending F_Registration signal and leaders update its routing table with followers' id, its link (leader) and their energy level.

If destination location is among follower nodes stop the grouping procedure.

Step 5: every follower node did not broadcast G_Request signal and nodes which receive that signal and a leader nor follower will respond with N_Response signal and search for destination node. 
If destination is found call corresponding follower node as mediator node by sending M_Select signal and Mediator sends L_Select signal to the destination node giving node_id=node_id +1 . Update its link and stop grouping procedure.

Else among the followers node, pick the node with highest energy level and farthest from leader, call it as mediator node by sending M_Select signal, here also ties are broken at random if two or more nodes have same energy level among followers.

Step 6: Mediator node broadcast G_Select signal. Nodes receiving that signal will send back its N_Response signal with residual energy level and node location. Mediator sends L_Select signal to the node that has highest energy level, calling it as leader assuming its node_id=node_id+1, ties are broken at random.

Step 7: Leader sends G_Request signal and nodes which receive that will respond with $\mathrm{N} \_$Response signal, the nodes which are not follower of any other group and responded declared as follower of that group by sending F Registration signal allocating node_id to the followers and update its routing table with follower's id, its link (leader) and their energy level. If destination location is among follower nodes stop the grouping procedure.

Step 8: Go to step 5, until either destination is found or all nodes are included in grouping or all nodes are dead.

In some cases, some of nodes may be not within range of any other nodes; they are as good as dead.

\subsubsection{The LFM algorithm to get LFM tree where} a mobile sink moves outside the boundary of restricted area, where sink is in direct link with at least one node located in the restricted area else move sink so as to get link with at least one node.

Input: Destination node location

Depending on the residual energy level of nodes, nodes are said to be in active (MODE 2), follower(MODE 1)or dead (MODE 0) mode.

Step 1: Sink broadcasts G_Request signal with Sink_id=1.

Step 2: Nodes receiving that signal sends N_Response signal.

Sink search for destination node.

If destination is found mark it with node_id=node_id +1 ,

Then stop grouping procedure.

Else go to step 3.

Step 3: Sink checks the residual energy of the responded nodes. If it is greater than or equal to upper threshold (MODE 2) level, then they are declared as leaders and marked with node_id=node_id +1 .

Step 4: Every leader node broadcast G_Request signal, Nodes receiving that signal sends $\mathrm{N}$ Response signal and leaders search for destination node.

If destination is found mark it with node_id=node_id+1 by sending F_Registration signal, update routing table, stop grouping procedure.
Else among leader nodes having one with highest energy level is taken as leader for further clustering. Ties are broken at random

Step 5: Selected Leader sends G_Request signal, Nodes receiving that signal sends $N_{-}$Response signal, the leader declares them as followers of that group by sending F_Registration signal giving node_id to its followers of that group and update its routing table with followers id , its link (leader) and their energy level. Repeat this process for all remaining leaders.

Step 6 : Every follower node broadcast G_Request signal, Nodes receiving that signal sends N_Response signal and search for destination node.

If destination is found call that follower node as mediator node and destination as leader by sending L_Select signal. update its routing table, stop grouping procedure.

Else among the followers node, pick the node with highest energy level and farthest call it as mediator node, by sending M_Select signal, here also ties are broken at random.

Step 7: Mediator node broadcast G_Request signal and nodes receiving that signal will send N_Response Signal . The node responded with highest energy level becomes leader and mediator sends L_Select signal, giving id to that node, ties are broken at random

Step 8: Leader sends G_Request signal, Nodes receiving that signal sends N_Response signal and leader search for destination node.

If destination is found marke that node with node_id by sending F_select signal, update its routing table, stop grouping procedure.

Else the nodes which are not follower of any other group but within range of the leader is declared as follower of that group by sending F_select signal allocating id to its followers and update its routing table with followers id, its link (leader) with their energy level.

Step 9: Go to step 6, until either destination is found or all nodes are included in grouping or all nodes are dead.

In some cases, some of nodes may be not within range of any other nodes; they are as good as dead.

\subsubsection{Path finding procedure with updating of energy of nodes, in the case where both sink and source in controlled area}

//path $[\ldots . .$.$] is an Array$

Step 1: If source is not a Leader

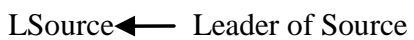

Else

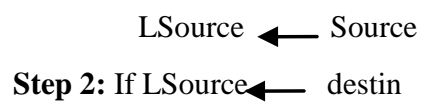

Place destin and source in path array

Calculate discharge of source and destin in data transfer process. 
Update energy of source and destin nodes

Else

Place destin in path array. Calculate discharge of destin in data transfer process.Update energy of destin nodes

If destin is not a leader

Ldest Leader of destin

Place Ldest in path array. Calculate discharge of Ldest in data transfer process.

Update energy of Ldest nodes

Else

\section{Ldest $\longleftarrow$ destin}

Step 3: While (Ldest is not equal LSource)

if Ldest is leader

Linc $\longleftarrow$ node linking Ldest to other group leader

Place Linc in path array. Calculate discharge of Ldest and

Linc in data transfer process. Update energy of Ldest and

Linc nodes

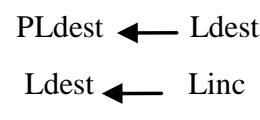

If (Ldest! =source)

Place source in path array. Calculate discharge of source source

and Ldest in data transfer process. Update energy of

and Ldest nodes

Path finding procedure with updating of energy of nodes, in the case where sink is mobile and outside restricted area may be written in the same way.

The LFM algorithm to get LFM tree where sink is moving outside the boundary of restricted area, is simulated using $\mathrm{C}++$ code, where grouping process starts from sink and results are shown in section 5 .

\section{DESIGN}

\subsection{State -Transition Diagram [12] of Nodes}

Initially Status is 0 (Reset)

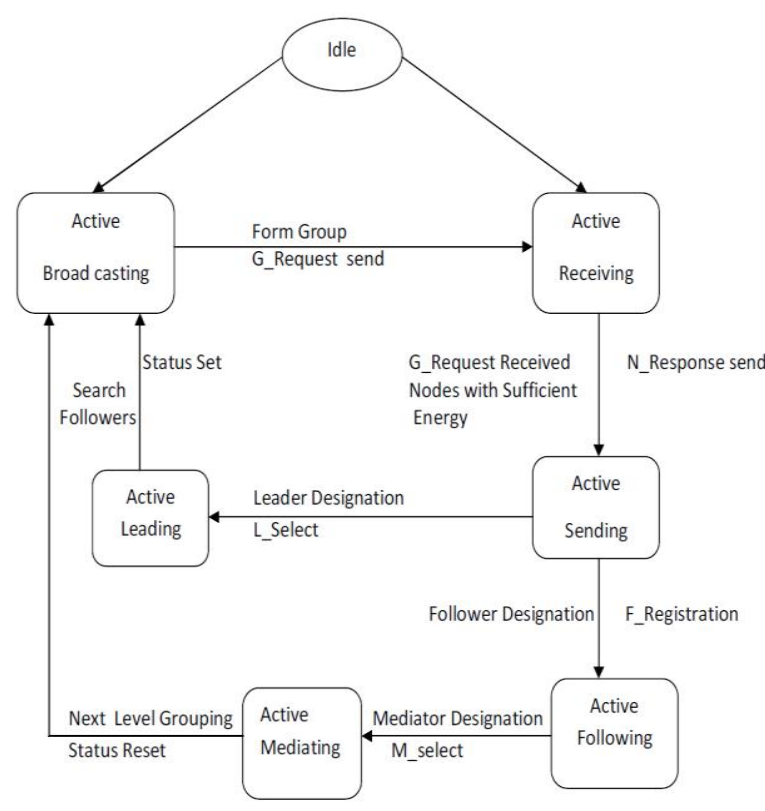

\section{IMPLEMENTATION OF CLUSTERING SCENARIO}

An instance of 30 nodes deployed in a rectangular controlled area is considered for simplicity. For testing the working of the designed grouping algorithm, it is implemented using $\mathrm{C}++$ codes .The data sets required are

1) Location of the nodes in the controlled area ( $x$ coordinate and y coordinate of nodes).

\subsection{Flow chart}

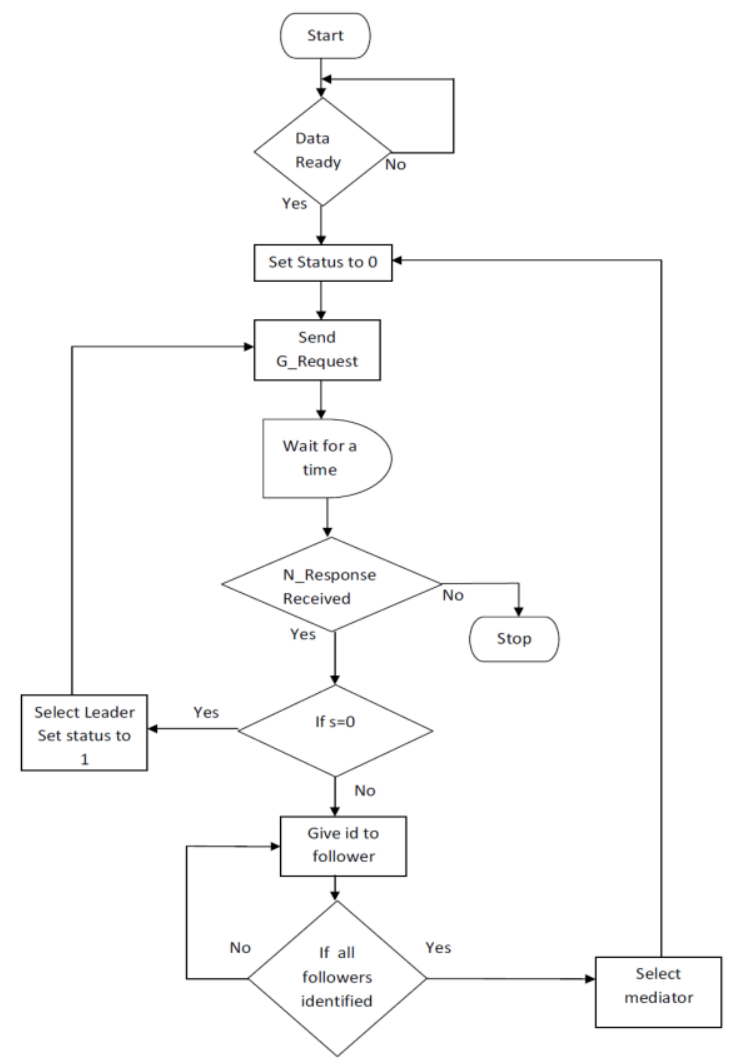


2) Sink node and destination node.

3) Initial residual energy to all nodes.
To make implementation easier, it is assumed that nodes are deployed in specific location so that its location is known and node_id is given to support grouping.

\subsection{Snapshots of LFM tree where sink is outside boundary area}

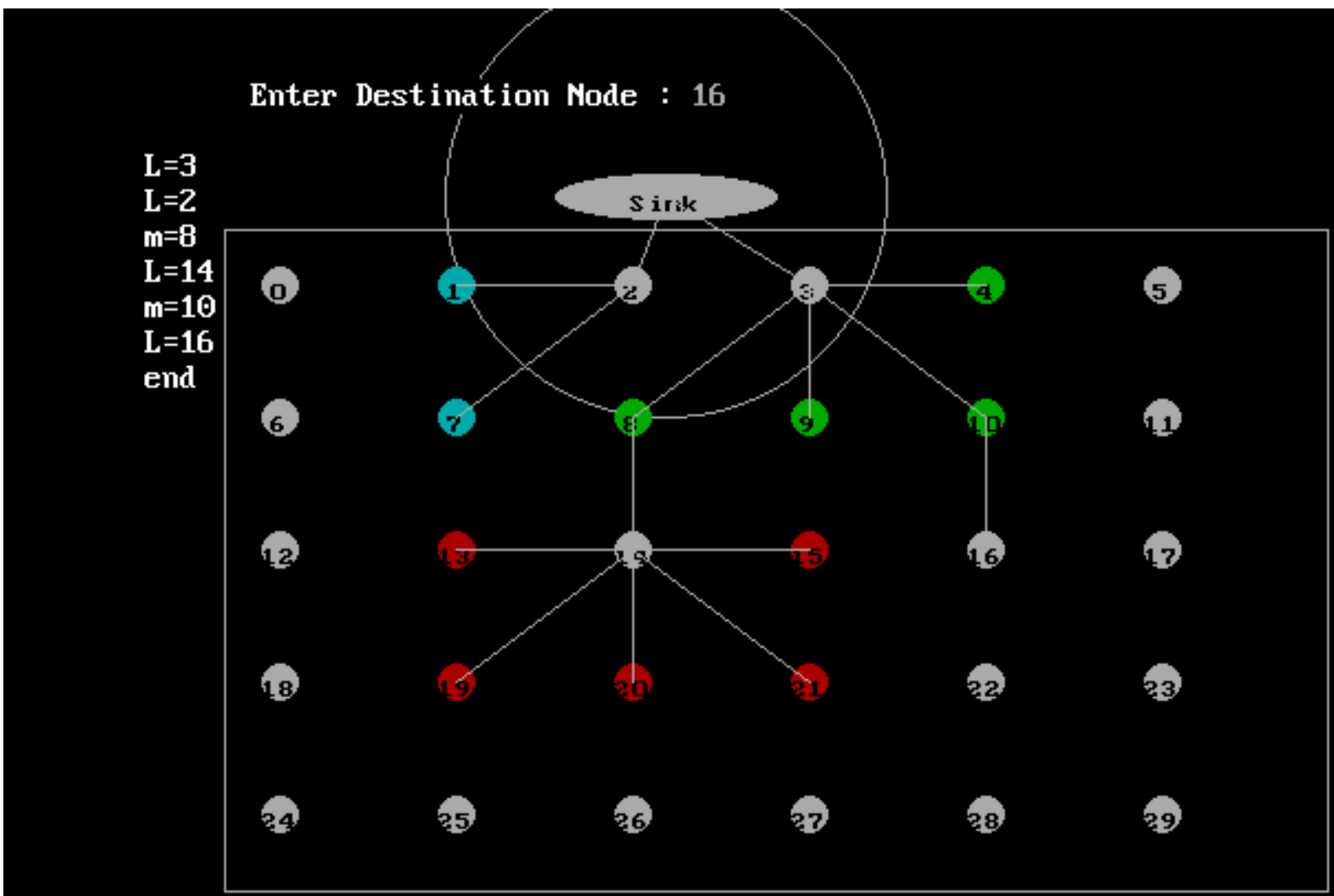

$\begin{array}{lll}\text { Leaders } & \text { Link } & \text { Followers } \\ 2 & -1 & ->7->1 \\ 3 & -1 & ->10->9->8->4 \\ 14 & 8 & ->21->20->19->15->13 \\ 16 & 10 & \end{array}$

PATH: Sink - 3-10-16

Fig 1 : Snapshot of Grouping from Sink node to node 16 with Routing information 
Enter Destination Mode : 16

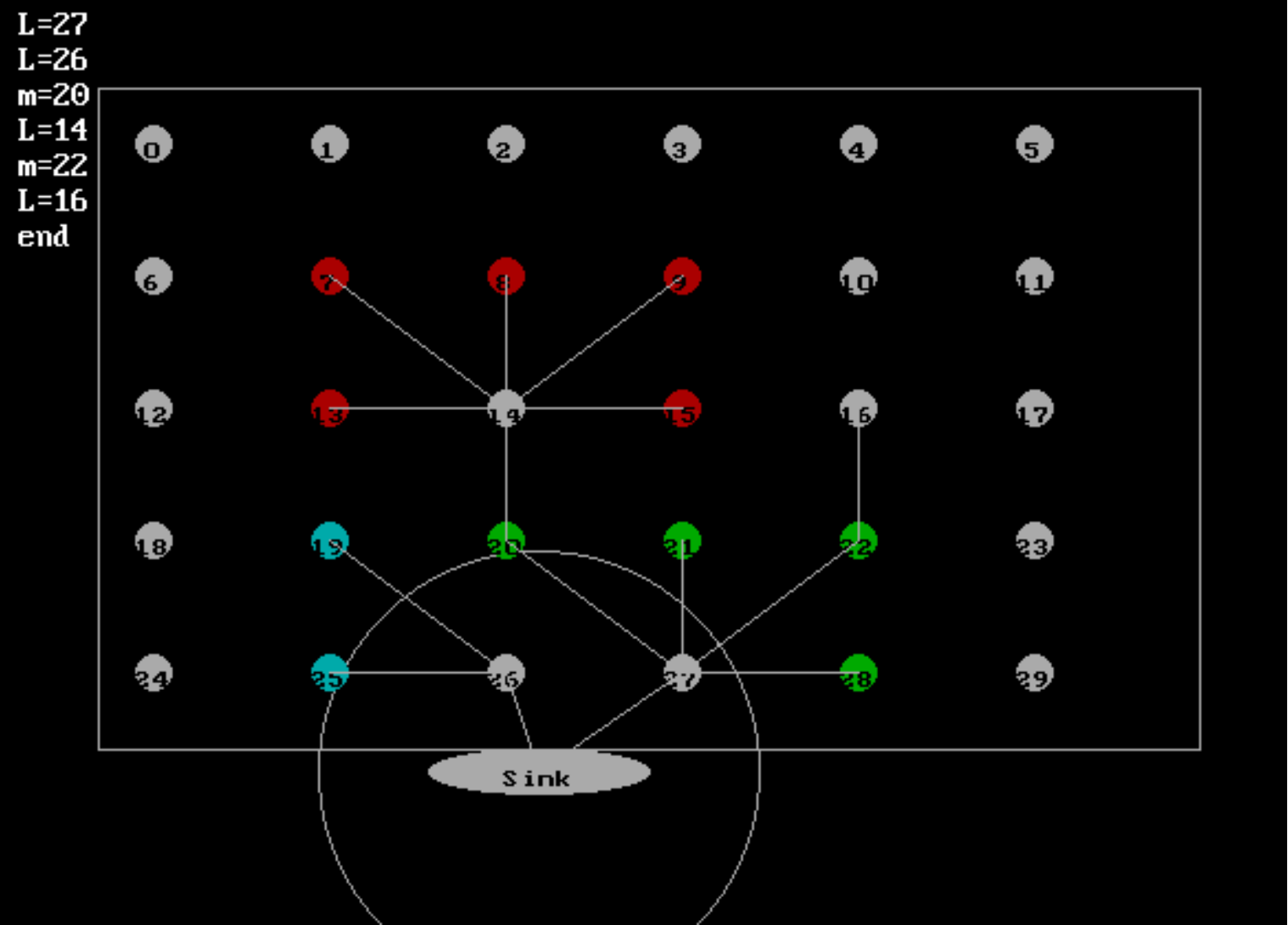

$\begin{array}{lll}\text { Leaders } & \text { Link } & \text { Followers } \\ 14 & 20 & ->15->13->9->8->7 \\ 16 & 22 & \\ 26 & -1 & ->25->19 \\ 27 & -1 & ->28->22->21->20\end{array}$

PATH: SINK $-27-22-16$

Fig 2: Snapshot of Next instance with Routing information when sink moved to other location

\subsection{Simulated results \& observations}

The grouping algorithm designed is implemented using $\mathrm{C}++$ codes and result of observation is plotted. The instance verses energy discharge is plotted shown in Fig.3. For simplicity we have considered nine instances. It has been observed that energy consumed by a particular node at the end of nine instances is less in mobile sink than static sink because as sink position changes particular node is not close to sink. Hence discharge of battery of nodes is more uniform, hence network life time is improved.

Fig.4 is plot of Instance verses Mode. While implementing our algorithm, we assumed node may fall into any one of three modes, ie Nodes with energy level below minimum threshold cannot participate in data communication is Mode 0 , Nodes with energy level above minimum threshold but less than some higher threshold value which can participate in data communication but can become only member of cluster is Mode 1, Nodes with energy level equal or above higher threshold value which can participate in data communication can become cluster head is Mode 2.It is observed that in case of mobile sink the nodes will continue in either in Mode2 or Mode 2 for longer duration because battery discharge is uniform in mobile sink scenario. 


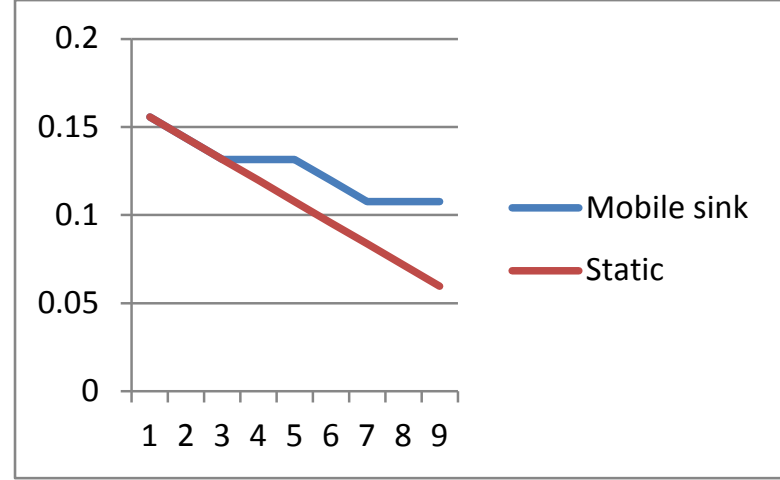

Instance

Fig.3 Instance verses Energy discharge graph

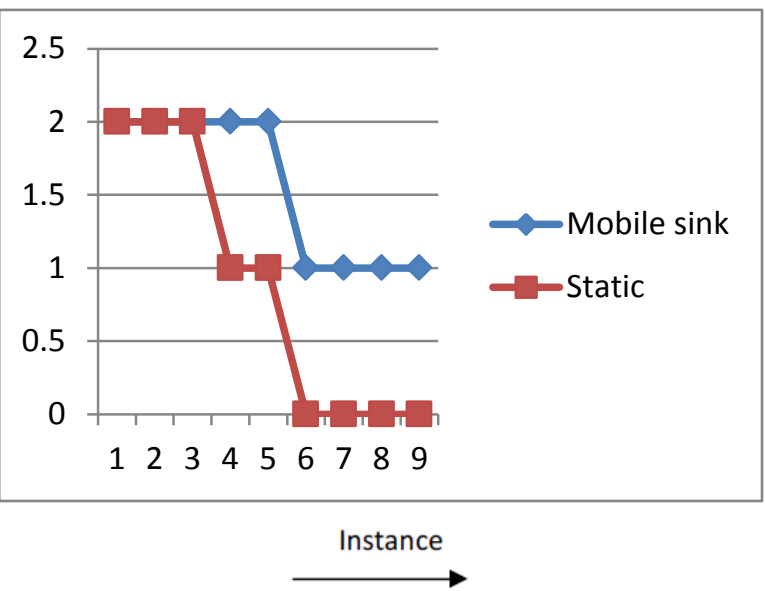

Fig.4 Instance verses Mode graph

\section{CONCLUSIONS}

One of the important observation is mobility of nodes can increase life time of the network. Concerning the routing of data from a node in question, the proposed LFM algorithm is efficient (number of hops wise, complexity wise, and power consumption wise), because of a tree data structure there by path to any node to any other node are unambiguous. Selection of mediator nodes in clustering avoids possibility of over lapping leaders there by number of groups formed will be less compared to existing clustering algorithms.

The proposed protocol is on demand protocol (Reactive) in which clustering is formed when a node has sensed data to send to other node and thus reduces the power needs of the nodes. Cluster members are decided at the instant not in advance as in proactive type. Particularly when nodes are in random motion at different rates, we need such dynamic grouping algorithms.

In sensor networks, nodes have no global identification problem which can be solved giving address to nodes while forming clusters as given in our LFM algorithm.

The limited resources of sensor nodes dictates that power is conserved and amount of data to be stored is limited, which is facilitated in the algorithm proposed by initiating the clustering from the source and stopping it as soon as the destination becomes either head or member of any group and clustering process checks whether incoming node in to grouping procedure is destination, thereby only the required nodes are clustered and other nodes are untouched. Only path from source to destination is to be stored.

Clustering also supports for media access control, hence cross layer optimization is achieved.

We may conclude that proposed LFM protocol is well suited for a network which uses memory hungry devices like sensor nodes.

The LFM protocol may be simulated using standard simulator like Network Simulator Ns2 and results may be compared with other routing protocols.

\section{REFERENCES}

[1] Marzieh Veyseh, Belle Wei and Nader F.Mir, An Information Management Protocol to control Routing and Clustering in Sensor Networks, Journal of computing and information Technology - CIT 13,2005, $1,53-68$

[2] R.Nagpal, Daniel Coore, AI Memo 1026, MIT 1997 An algorithm for group formation in Amorophous Computer

[3] Saad Ahmed Munir, Biao Ren, Weiwei Jiao, Bin Wang, Dongliang Xie, Jian Ma Mobile Wireless Sensor Network: Architecture and Enabling Technologies for Ubiquitous Computing IEEE 21st International Conference on Advanced Information Networking and Workshops (AINAW'07) 0-7695-2847-3/07 \$20.00 (c) 2007.

[4] I.F.Akyldiz, W.Su etal, A Survey on Sensor Networks,IEEE Communications Magazine, Vol.40,Issue 8,August 2002

[5] Nader F. Mir, "Computer and Communication Networks", Pearson Education, 2007.

[6] Laiali Almazaydesh,Eman Abdelfattah,Manal ALBzoor and Amer Al-Rahayfch, Performance Evaluation of Routing Protocols in Wireless Sensor Networks, International Journal of Computer Science and Information Technology,Volume 2,Number 2,April 2010.

[7] Jichuan Zhao,Ahmet T. Erdogan and Tughrul Arslan, A Novel Application Specific Network Protocol For Wireless Sensor Networks,IEEE 2005.

[8] W.RabinerHienzelman,Ananth Chandrashekaran and Hari Balakrishna, Energy Efficient Communication Protocol For Wireless Sensor Networks, Hawaii International Conference on system sciences Jan 4-7 2000 .

[9] Bandyopadhyay, S and coyle, E.J.,An energy efficient hierarchical clustering algorithm For Wireless Sensor Networks, Twenty second Annual Joint Conf. of the IEEE Computer and Commun.Soc., Vol.3, pp 17131723,April 2003.

[10] H"useyin Akcan CIS Department Polytechnic University GPS-Free Node Localization in Mobile Wireless Sensor Networks in MobiDE'06, June 25, 2006, Chicago, Illinois, USA2006 ACM 1

[11] Lei Wang 1 and Qingzheng Xu GPS-Free Localization Algorithm for Wireless Sensor Networks 1,2 Sensors 
2010, 10, 5899-5926; doi:10.3390/s100605899 sensors ISSN $1424-8220$

[12] Pallapa Venkataram,Sunil kumar S.Manvi Communication Protocol Publication,New Delhi 2004.

\section{AUTHORS PROFILE}

Venkatramana Bhat $\mathbf{P}$. is currently the Professor and HOD, Department of Computer Science Engineering, Vivekananda College of Engineering Technology, and Puttur (D.K) affiliated to VTU, Belgum. He obtained his Bachelor of Engineering from Mysore University. He received his Masters degree in Computer Science and Engineering from Manipal Institute of Technology, Manipal and presently pursuing Ph.D. in Computer Science and Engineering under Manipal University.

Dr.Shridhar Aithal, Professor T.A. Pai Management Institute Manipal, having tweleve Years of Industrial Experience and fourteen years of Academic experience .He is also approved Research Guide for Manipal University Manipal.His subject of intrest mainly includes Wireless Sensor Network, Software Engineering,Management Information System.

Dr.Sudhakar Adiga, Professor, Department of Mathematics having 25 Years of academics experience, did his Ph.D in Graph Theory and working in Algebraic graph Theory.His area of interest includes Combinatorics. 